\title{
LIVER
}

\section{Bilirubin inhibits bile acid induced apoptosis in rat hepatocytes}

\author{
A Granato, G Gores, M T Vilei, R Tolando, C Ferraresso, M Muraca
}

See end of article for authors' affiliations

.....................

Correspondence to: Dr M Muraca, Department of Medical and Surgical Sciences, University of Padova, Via Giustiniani, 2, I-35128 Padova, Italy; muraca@unipd.it

Accepted for publication 25 June 2003
Background and aims: Hydrophobic bile acids contribute to hepatocellular injury in cholestasis and rapidly induce apoptosis in vitro; however, unlike Fas agonists, cholestasis does not cause extensive hepatocyte apoptosis. As antioxidants provide protection against bile acid induced liver injury, our premise was that bilirubin, a free radical scavenger with increased plasma levels in the presence of liver disease, could protect hepatocytes against bile acid induced apoptosis.

Methods: Freshly isolated rat hepatocytes were incubated for four hours with $100 \mu \mathrm{mol} / \mathrm{l}$ glycochenodeoxycholate (GCDC) alone or with increasing concentrations of unconjugated (UCB) or conjugated (CB) bilirubin.

Results: Both UCB and CB inhibited GCDC induced apoptosis in a dose dependent fashion and suppressed the generation of reactive oxygen species by hepatocytes.

Conclusions: The antiapoptotic effect of bilirubin associated with its antioxidant properties indicates that hyperbilirubinaemia may have a protective role in liver disease. $\mathrm{n}$ patients with cholestasis, accumulation of bile acids within the liver contributes to hepatocellular damage. While high bile acid concentrations can induce hepatocyte necrosis, lower concentrations of these compounds are associated with apoptosis ${ }^{12}$ which can be triggered by specific cell surface "death" receptors (extrinsic pathway) or by mitochondrial dysfunction induced by internal stress (intrinsic pathway), ${ }^{3}$ such as the generation of reactive oxygen species (ROS)..$^{4-6}$

Despite an increasing body of evidence demonstrating that bile acid mediated hepatocyte apoptosis plays an important role in hepatobiliary diseases, ${ }^{3}{ }^{7}$ the incidence of parenchymal liver cell apoptosis in cholestatic disorders is lower than expected on the basis of in vitro studies. ${ }^{8}$ Possible explanations for this discrepancy include activation of antiapoptotic pathways $^{89}$ and the presence of protective humoral factors. Free radical scavengers such as lazaroids, ${ }^{10} \alpha$-tocopherol, and ebselen ${ }^{4}$ inhibit bile acid induced hepatocyte apoptosis in vitro. Bilirubin, a yellow tetrapyrrole derived from the enzymatic degradation of haeme, also accumulates in the plasma of patients with cholestasis due to impaired biliary excretion. ${ }^{11}$ This lipid soluble pigment is commonly considered merely a toxic waste compound because neonatal hyperbilirubinaemia can lead to brain damage ("kernicterus" ${ }^{\prime}{ }^{1}$ However, the pigment acts as an efficient free radical scavenger in vitro at micromolar concentrations. ${ }^{13-15}$ Prior to biliary excretion, bilirubin is conjugated with one or two molecules of glucuronic acid, yielding a water soluble compound. Unconjugated (UCB) and conjugated (CB) bilirubin accumulate in tissues and plasma during cholestasis. ${ }^{11} 16$

In the present work, we demonstrate that both UCB and CB effectively inhibit bile acid induced apoptosis in freshly isolated hepatocytes, an effect associated with suppression of ROS generation, suggesting that these pigments may play a protective role in cholestatic disorders.

\section{MATERIALS AND METHODS} Materials

CD rats were purchased from Charles River (Kisslegg, Germany). William's E medium, fetal bovine serum, peni- cillin/streptomycin, glutamine, and collagenase were from Life Technologies Inc. (Grand Island, New York, USA). Trypan blue, dexamethasone, glucagon, glycochenodeoxycholate (GCDC), diamidino-2-phenylindole dihydrochloride (DAPI), and propidium iodide were supplied by Sigma Chemical Co. (St. Louis, Missouri, USA). UCB (Sigma Chemical Co) was recrystallised before use. ${ }^{17} \mathrm{CB}$ ditaurate (a commercially available surrogate for bilirubin glucuronate) was from Porphyrin Products Inc. (Logan, Utah, USA). According to the manufacturer, the pigment contains approximately $10-12 \%$ monotaurate and less than $2 \%$ of UCB.

\section{Experimental methods \\ Preparation of stock solutions of $U C B$ and $C B$}

Stock solutions of 42.75, 21.38, and $10.69 \mathrm{mmol} / \mathrm{l}$ UCB were prepared by dissolving 25, 12.5, and $6.25 \mathrm{mg}$ of pigment, respectively, in $1 \mathrm{ml}$ of $0.1 \mathrm{~N} \mathrm{NaOH}$. After complete dissolution, $1.750 \mathrm{ml}$ of albumin solution (70 $\mathrm{mg}$ bovine serum albumin (BSA) in $1.75 \mathrm{ml}$ of William's E medium) were added to each UCB solution. The solutions, constantly protected from light, were diluted with incubation medium containing $5 \%$ fetal bovine serum to the desired pigment concentrations $(200,100$, and $50 \mu \mathrm{mol} / \mathrm{l})$ just before use. As the albumin content of $5 \%$ fetal bovine serum corresponded to $13.44 \mu \mathrm{mol} / \mathrm{l}$, the final albumin concentration in incubation medium $(4.73 \mu \mathrm{mol} / \mathrm{l}$ from added BSA+13.44 $\mu \mathrm{mol} / \mathrm{l}$ from fetal bovine serum) was $18.17 \mu \mathrm{mol} / \mathrm{l}$. The bilirubin/ albumin molar ratios in the 200, 100, and $50 \mu \mathrm{mol} / \mathrm{l}$ solutions were therefore 11, 5.5, and 2.75, respectively. As bile salts are also bound to albumin, the albumin solution without bilirubin was diluted with incubation medium to obtain the same final concentration of BSA in experiments not including bile pigments. The GCDC/albumin molar ratio was 5.5.

Abbreviations: GCDC, glycochenodeoxycholate; UCB, unconjugated bilirubin; $C B$, conjugated bilirubin; ROS, reactive oxygen species; $D A P I$ diamidino-2-phenylindole dihydrochloride; BSA, bovine serum albumin; DCF, dichlorofluorescein; LDH, lactate dehydrogenase; GLDH, glutamate dehydrogenase; AST, aspartate aminotransferase 
UCB solution was centrifuged and the bilirubin concentration assessed in duplicate by the diazo method before and after centrifugation. No change in bilirubin concentration was observed after centrifugation, nor were pigment aggregates found at microscopic examination $(400 \times)$ of the solution. The $\mathrm{pH}$ of the culture medium containing BSA-UCB $(10 \mathrm{ml}$ aliquots) was adjusted to 7.4 by adding $10-25 \mu \mathrm{l}$ of $1.2 \mathrm{M}$ $\mathrm{HCl}$. CB was dissolved directly in William's E medium containing BSA and fetal bovine serum as described above, and no $\mathrm{pH}$ correction was necessary.

\section{Hepatocyte isolation and culture}

Rat primary hepatocytes were isolated from male CD rats $(150-200 \mathrm{~g})$ by collagenase perfusion of the liver. ${ }^{18}$ Cell viability, determined by trypan blue exclusion, was $85-93 \%$. After isolation, hepatocytes were resuspended in William's E medium supplemented with $10 \%$ fetal bovine serum, $100 \mathrm{U} / \mathrm{ml}$ penicillin, $100 \mathrm{U} / \mathrm{ml}$ streptomycin, $3 \mathrm{mM}$ glutamine, $0.16 \mathrm{U} /$ $\mathrm{ml}$ insulin, $9.6 \mu \mathrm{g} / \mathrm{ml}$ dexamethasone, and $0.014 \mu \mathrm{g} / \mathrm{ml}$ glucagon. A total of $5 \times 10^{5}$ cells/well, seeded onto uncoated plastic tissue culture plates, were maintained at $37^{\circ} \mathrm{C}$ in a $5 \% \mathrm{CO}_{2}$ humidified atmosphere for three hours. Plates were then washed with the medium to remove unattached cells and incubated with a medium containing 5\% fetal bovine serum, $100 \mathrm{U} / \mathrm{ml}$ penicillin, $100 \mathrm{U} / \mathrm{ml}$ streptomycin, $3 \mathrm{mM}$ glutamine, $0.16 \mathrm{U} / \mathrm{ml}$ insulin, and $100 \mu \mathrm{mol} / \mathrm{l} \mathrm{GCDC}$, alone or in combination with 50,100 , or $200 \mu \mathrm{mol} / \mathrm{l} \mathrm{UCB}$ or CB for four hours at $37^{\circ} \mathrm{C}$ in $5 \% \mathrm{CO}_{2}$ humidified atmosphere. Elsewhere, we observed that approximately $1 \%$ of UCB added to the incubation medium is converted by cultured rat hepatocytes to $\mathrm{CB}$ after four hours. ${ }^{19}$

Assessment of nuclear fragmentation (apoptosis) Isolated rat hepatocytes were plated onto uncoated plastic tissue culture plates $\left(5 \times 10^{5}\right.$ cells/well) and incubated for four hours with $100 \mu \mathrm{mol} / \mathrm{l} \mathrm{GCDC}$ alone or in combination with UCB or CB. Nuclear changes indicating apoptosis were quantified by staining rat hepatocytes with DAPI, a membrane permeant fluorescent DNA binding dye to label the nucleus of hepatocytes. Propidium iodide $(1 \mu \mathrm{M})$ was also added to identify necrotic cells. For morphological evaluation of apoptosis at fluorescence microscopy, cultured rat hepatocytes were incubated with DAPI ( $1 \mu \mathrm{g} / \mathrm{ml})$ for 10 minutes at $37^{\circ} \mathrm{C}$. Cells were considered apoptotic if the classic features of nuclear margination/condensation and nuclear fragmentation were present. Fluorescent stained nuclei were considered fragmented if at least three separate fragments of condensate chromatin were identified in a cell. At least 300 cells in four high power fields were counted, and nuclear fragmentation was expressed as a percentage of total cells, excluding propidium iodide. $^{2021}$

\section{Measurement of reactive oxygen species (ROS) generation}

Isolated hepatocytes $\left(5 \times 10^{5}\right.$ cells/well $)$ were preloaded with $10 \mu \mathrm{mol} / \mathrm{l} 2.79$-dichlorofluorescein (DCF) at $37^{\circ} \mathrm{C}$ for $30 \mathrm{~min}$ utes, washed twice, and resuspended in the incubation medium with or without $100 \mu \mathrm{mol} / 1$ GCDC and in the presence or absence of 50, 100, and $200 \mu \mathrm{mol} / \mathrm{l} \mathrm{UCB}$ or CB. Aliquots of cells, removed at 0, 60, 120, 180, and $240 \mathrm{~min}-$ utes, were analysed for fluorescence in real time at $490 \mathrm{~nm}$ excitation and $520 \mathrm{~nm}$ emission wavelengths in a 1420 Victor 2 (EG\&G Wallac, Turku, Finland) fluorescence spectrophotometer.

\section{Enzyme release}

During the four hour incubation of hepatocytes with or without GCDC, UCB, and CB, cellular release of lactate dehydrogenase (LDH), glutamate dehydrogenase (GLDH), and aspartate aminotransferase (AST) was measured hourly, and expressed as a percentage of enzyme released into the buffer of the total activity present in hepatocytes (sum of the activities measured after cell lysis and in the medium). Measurement of GLDH, LDH, and AST was achieved using enzymatic method kits (Randox, Crumlin, UK, Roche Diagnostica SpA, Monza, Italy, and Roche Diagnostica SpA, Monza, Italy, respectively). All measurements were performed using an automatic analyser (Hitachi 912, Tokyo, Japan).

\section{Statistics}

Values are expressed as mean (SD). A GraphPad InStat microcomputer program (GraphPad Software, Inc. San Diego, California, USA) was used to evaluate differences between groups with the Mann-Whitney rank sum test and regression analyses. A $p$ value $<0.05$ was considered statistically significant.

\section{RESULTS}

\section{Morphological features of apoptosis}

After four hours of treatment with $100 \mu \mathrm{mol} / \mathrm{l} \mathrm{GCDC}, 63.32 \%$ ( $1.84 \%$ ) of hepatocytes were apoptotic whereas $<1 \%$ of cells were apoptotic in the absence of the bile acid, as assessed by nuclear fragmentation after DAPI staining (table 1 , fig 1 ).

Table 1 Effect of unconjugated (UCB) and conjugated (CB) bilirubin on glycochenodeoxycholate (GCDC) induced apoptosis. Rat primary hepatocytes in culture were incubated for four hours with incubation medium alone or with $100 \mu \mathrm{mol} / \mathrm{I} \mathrm{GCDC}$ and with increasing concentrations of $U C B$ and $C B$

\begin{tabular}{lc}
\hline Experimental group $(\mathbf{n}=6)$ & \% Apoptosis \\
\hline Incubation medium & $0.66(0.51)$ \\
UCB $50 \mu M$ & $0.67(0.52)$ \\
UCB $100 \mu M$ & $0.83(0.41)$ \\
UCB $200 \mu M$ & $0.66(0.51)$ \\
CB $50 \mu M$ & $0.83(0.41)$ \\
CB $100 \mu M$ & $0.70(0.54)$ \\
CB $200 \mu M$ & $0.70(0.55)$ \\
GCDCA $100 \mu M$ & $63.32(1.84)$ \\
GCDCA $100 \mu M+U C B ~ 50 \mu M$ & $55.97(3.28)^{* *}$ \\
GCDCA $100 \mu M+U C B ~ 100 \mu M$ & $42.92(2.91)^{* *}$ \\
GCDCA $100 \mu M+U C B ~ 200 \mu M$ & $38.21(2.15)^{* *}$ \\
GCDCA $100 \mu M+C B ~ 50 \mu M$ & $52.50(2.43)^{* *}$ \\
GCDCA $100 \mu M+C B ~ 100 \mu M$ & $35.50(3.08)$ \\
GCDCA $100 \mu M+C B ~ 200 \mu M$ & $30.33(4.27)$ \\
\hline
\end{tabular}

Results are expressed as mean (SD) per cent apoptosis occurring in GCDC treated cells. ${ }^{\star *} \mathrm{p}<0.005$ compared with GCDC. 


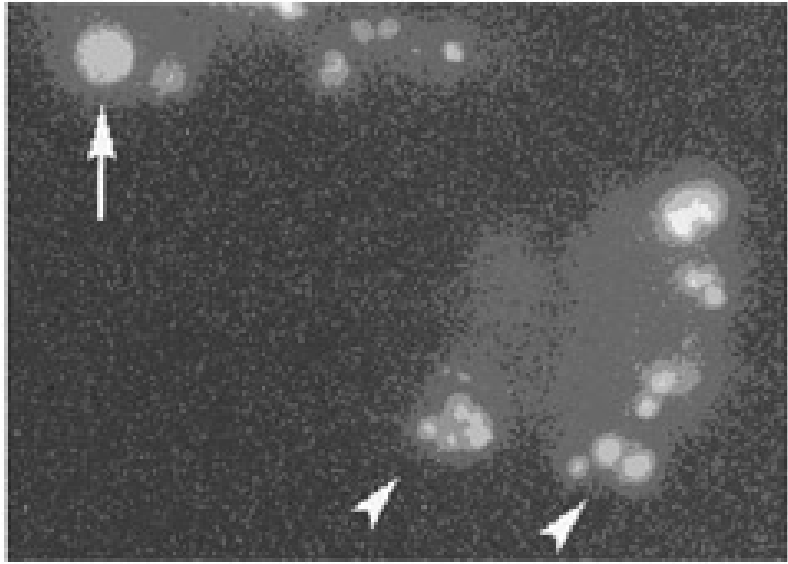

Figure 1 Morphological features of glycochenodeoxycholate (GCDC) induced apoptosis of rat hepatocytes. Fluorescence photomicrograph of cells stained with the DNA binding dye diamidino-2-phenylindole dihydrochloride (DAPI). Cells undergoing apoptosis after four hours of incubation with $100 \mu \mathrm{mol} / / \mathrm{GCDC}$ showed nuclear fragmentation (arrowheads). A cell with a normal nucleus is visible on the upper left corner (arrow) (original magnification 400x).

Both UCB and CB inhibited GCDC induced apoptosis in a concentration dependent fashion. The inhibitory effect of $\mathrm{CB}$ on GCDC induced apoptosis was significantly stronger compared with UCB (fig 2).

\section{GCDC induced ROS generation}

In order to investigate any relationship between bilirubin inhibition of GCDC induced apoptosis and the antioxidant properties of the pigment, ROS generation was assessed in rat hepatocytes treated with $100 \mu \mathrm{mol} / \mathrm{l} \mathrm{GCDC}$ in the presence or absence of 50, 100, and $200 \mu \mathrm{mol} / \mathrm{l} \mathrm{UCB}$ or CB, as described above. Addition of both pigments strongly suppressed the increase in GCDC stimulated DCF fluorescence, thus indicating strong suppression of ROS generation (figs 3, 4; only data obtained at a concentration of $100 \mu \mathrm{mol} / \mathrm{l}$ are shown). Inhibition of ROS generation was not significantly different with UCB or CB at any concentration tested.

\section{Enzyme release}

Incubation with $100 \mu \mathrm{mol} / \mathrm{l} \mathrm{GCDC}$ was followed by increased release of LDH, GLDH, and AST with respect to control

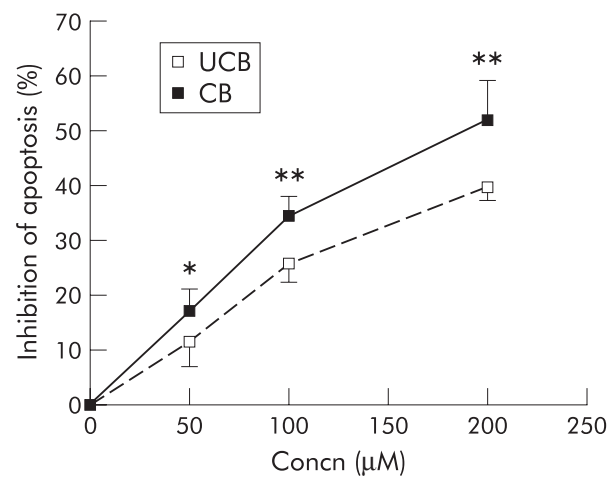

Figure 2 Inhibition of glycochenodeoxycholate (GCDC) induced apoptosis by unconjugated (UCB) and conjugated (CB) bilirubin. Rat primary hepatocytes in culture were incubated for four hours with $100 \mu \mathrm{mol} / /$ GCDC and with increasing concentrations of UCB or CB. Both the unconjugated and conjugated pigment inhibited GCDC induced apoptosis but $C B$ exhibited a stronger effect (mean (SD), $n=6$ ). ${ }^{*} p<0.05,{ }^{* *} p<0.005$ compared with UCB.

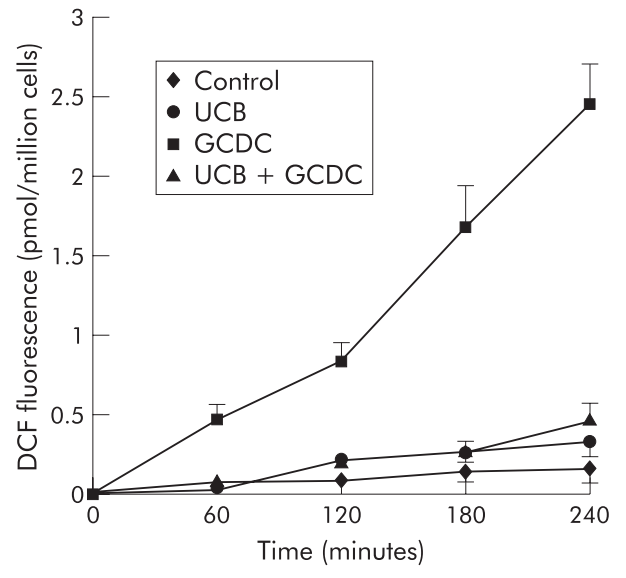

Figure 3 Effect of unconjugated bilirubin (UCB) on glycochenodeoxycholate (GCDC) induced hydroperoxide generation. Rat hepatocytes were incubated for four hours with control culture medium, or with medium containing $100 \mu \mathrm{mol} / \mathrm{I} U C B, 100 \mu \mathrm{mol} / \mathrm{I}$ GCDC, or a combination of both. UCB strongly suppressed GCDC induced 2.79-dichlorofluorescein (DCF) fluorescence, a measurement of hydroperoxide generation (mean (SD), $n=6$ ).

samples. Coincubation with $100 \mu \mathrm{mol} / \mathrm{l}$ UCB or CB strongly reduced the release of all enzymes tested (figs 5, 6).

\section{DISCUSSION}

Although our understanding of the pathogenesis of cholestatic liver disease is incomplete, it is generally believed that accumulation of toxic hydrophobic bile acids, such as deoxycholic acid conjugates, within the hepatocyte can contribute to liver injury by inducing hepatocyte apoptosis. ${ }^{73}$ Antioxidants, such as $\alpha$-tocopherol or lazaroid, reduce both the generation of ROS and cell injury in freshly isolated hepatocytes treated with $\mathrm{GCDC}^{4}{ }^{10}$ as well as in the intact rat infused with taurochenodeoxycholic acid. ${ }^{22}$ Bilirubin, the yellow pigment which accumulates in the plasma of patients with cholestasis, was recognised as an antioxidant of possible physiological importance approximately 15 years ago, and its activity as a free radical scavenger was demonstrated in model membrane systems, being equal to or even surpassing

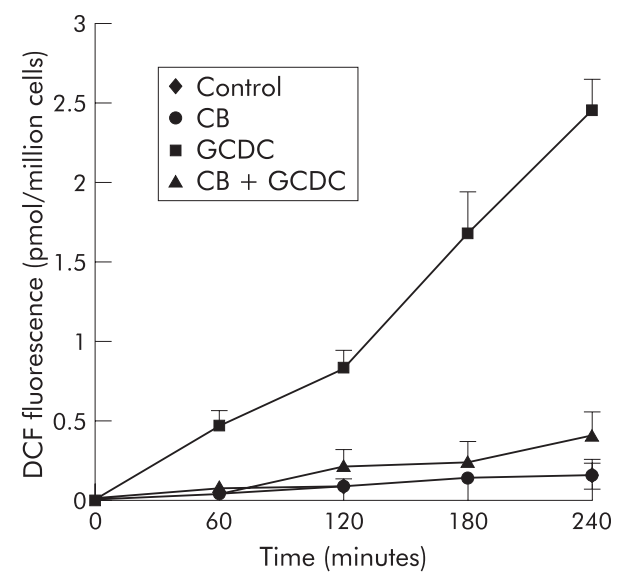

Figure 4 Effect of conjugated bilirubin (CB) on glycochenodeoxycholate (GCDC) induced hydroperoxide generation. Rat hepatocytes were incubated for four hours with control culture medium, or with medium containing $100 \mu \mathrm{mol} / \mathrm{I} \mathrm{CB}, 100 \mu \mathrm{mol} / \mathrm{I}$ GCDC , or a combination of both. CB strongly suppressed GCDC induced 2.79-dichlorofluorescein (DCF) fluorescence, a measurement of hydroperoxide generation (mean (SD), $n=6$ ). 

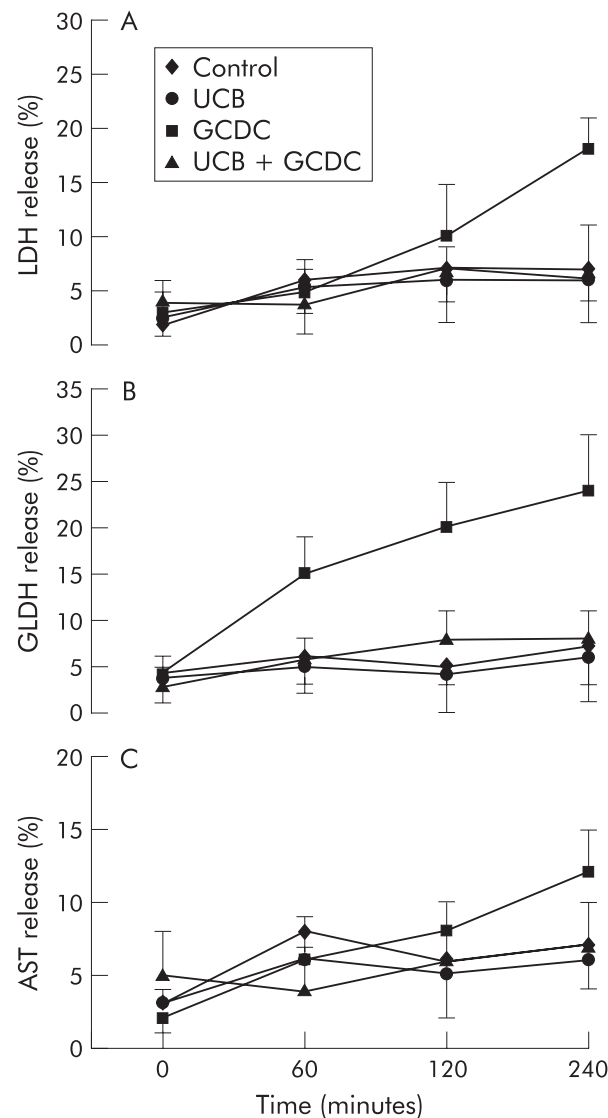

Figure 5 Bile acid stimulated enzyme release from hepatocytes was inhibited by unconjugated bilirubin (UCB). Hepatocytes were incubated with $100 \mu \mathrm{mol} / \mathrm{l}$ glycochenodeoxycholate (GCDC) with or without $100 \mu \mathrm{mol} / / \mathrm{I}$ UCB. Enzyme activity released in the incubation medium was expressed as per cent of total enzyme activity measured on cell lysates at the end of each time point. Release of lactate dehydrogenase $(\mathrm{LDH})(\mathrm{A})$, glutamate dehydrogenase $(\mathrm{GLDH})(\mathrm{B})$, and aspartate aminotransferase (AST) (C) to control medium and after incubation with UCB, GCDC, and GCDC+UCB for four hours (mean (SD), $n=6$ ).

that of $\alpha$-tocopherol. ${ }^{13}{ }^{14}$ As bilirubin interacts with biomembranes, it was postulated that this pigment could prevent lipid peroxidation associated with alterations in the physicochemical properties of the membranes leading to cell dysfunction and death. ${ }^{13}$ The protection against oxidative stress provided by the pigment has been demonstrated in several in vitro and in vivo studies. ${ }^{15} 2324$

The findings in the present study demonstrate that bilirubin inhibits GCDC induced apoptosis in rat hepatocytes, and that this effect is associated with inhibition of ROS generation in the same culture system, thus suggesting a link between the antioxidant properties of the pigment and its ability to prevent bile acid induced apoptosis. Four hour incubation of hepatocytes with GCDC led to a slight increase in $\mathrm{LDH}, \mathrm{GLDH}$, and AST, which was prevented by addition of bile pigments to the medium (figs 5, 6), further suggesting that the membrane protective activity of bilirubin could help to reduce bile acid induced cell death.

The range of bilirubin concentrations tested in the in vitro assays are commonly found in the plasma of patients with cholestatic disorders. In these patients, plasma concentrations of bile acids and bilirubin are about equimolar. ${ }^{25}$ The final BSA concentration in incubation medium, corresponding to $18.17 \mu \mathrm{mol} / \mathrm{l}$, was well below both physiological levels (approximately $600 \mu \mathrm{mol} / \mathrm{l}$ ) and levels observed in patients
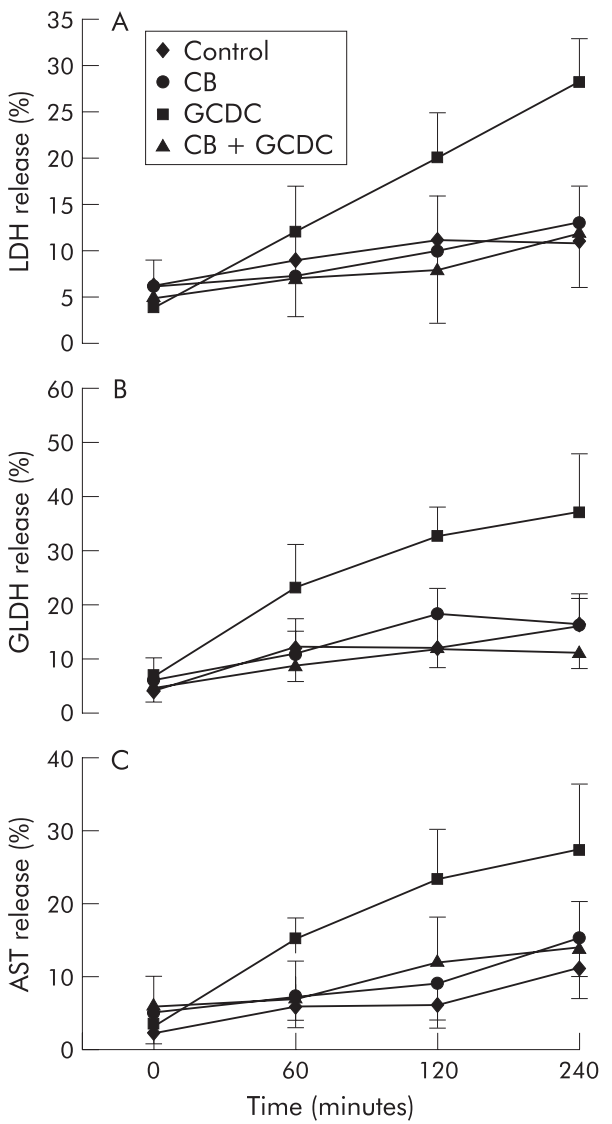

Figure 6 Bile acid stimulated enzyme release from hepatocytes was inhibited by conjugated bilirubin (CB). Hepatocytes were incubated with $100 \mu \mathrm{mol} / /$ glycochenodeoxycholate (GCDC) with or without $100 \mu \mathrm{mol} / \mathrm{I} \mathrm{CB}$. Enzyme activity released in the incubation medium was expressed as per cent of total enzyme activity measured on cell lysates at the end of each time point. Release of lactate dehydrogenase (LDH) (A), glutamate dehydrogenase (GLDH) (B), and aspartate aminotransferase (AST) (C) to control medium and after incubation with $C B, G C D C$, and $\mathrm{GCDC}+\mathrm{CB}$ for four hours (mean (SD), $\mathrm{n}=6$ ).

with end stage cholestatic liver disease (approximately $300 \mu \mathrm{mol} / \mathrm{l}$ ). However, low albumin concentrations have been used in all published studies involving apoptosis induced in vitro by toxic albumin bound compounds in order to achieve, in short incubation periods, significant interaction of the test compound with cultured cells, thus mimicking the conditions of a prolonged exposure in vivo (such as in cholestasis). ${ }^{124-626}$ Moreover CB, which is not bound to albumin and is the most abundant pigment fraction in the blood of cholestatic patients, inhibited bile acid induced apoptosis to an even greater extent than UCB (fig 2). Preliminary results showed that $\mathrm{CB}$ also behaves as a free radical scavenger. ${ }^{14}$ In the present experimental setup, suppression of ROS generation was similar with the two pigments. However, further studies are needed to compare the relative antioxidant effect of UCB and CB.

The above findings indicate that bilirubin may prevent the bile acid induced liver injury associated with cholestatic disorders, thus supporting the hypothesis that the pigment plays a "beneficial" role as a powerful biological antioxidant. ${ }^{13}{ }^{14}$ In our incubation system with freshly isolated hepatocytes, addition of $100 \mu \mathrm{mol} / \mathrm{l} \mathrm{UCB}$ or $\mathrm{CB}$ almost completely prevented GCDC induced ROS formation (figs 3, 4 ), but the average inhibition of apoptosis at the same pigment concentration was only $25.9 \%$ and $34.5 \%$, respectively. These data lend support to the hypothesis that 
oxidative stress is not the main trigger of apoptosis but is a secondary phenomenon, amplifying the toxic effect of bile acids. ${ }^{3}$

According to Silva and colleagues, ${ }^{26}$ UCB induced apoptosis in cultured neuronal cells, and this effect could be prevented by ursochenodeoxycholic acid. These results seem opposite to our findings. However, our experimental conditions are not comparable with those of Silva et al because while UCB is toxic to neurones it has not been shown to have any adverse effects on hepatocytes. Moreover, ursodeoxycholic acid is an antiapoptotic hydrophilic bile $\operatorname{acid}^{27}$ whereas GCDC is a detergent toxic compound.

In conclusion, our experimental findings indicate that the antioxidant properties of bilirubin may be relevant in liver disease, suggesting a protective role of the pigment accumulating in plasma and tissues of patients with cholestasis. The present results could have therapeutic implications as removal of bile pigments from plasma by liver support device ${ }^{28-30}$ might not be necessarily advantageous in patients with liver disease. As concentrations of UCB were in a similar range as serum total bilirubin levels in physiological jaundice, these findings suggest that physiological jaundice may also have antioxidant properties useful in the newborn. Further studies are needed to assess the clinical relevance of these findings.

\section{ACKNOWLEDGEMENTS}

This work was financed by Regione del Veneto, Giunta Regionale, Ricerca Sanitaria finalizzata n. 03/03/01, Venezia, Italy

\section{Authors' affiliations}

A Granato, M T Vilei, C Ferraresso, M Muraca, Clinica Medica 1, University of Padova, Italy

G Gores, Division of Gastroenterology and Hepatology, Mayo Medical

School, Clinic and Foundation, Rochester, Minnesota, USA

R Tolando, GlaxoSmithKline Research Centre, Verona, Italy

\section{REFERENCES}

1 Sokol RJ, Winklhofer-Roob BM, Devereaux MW, et al. Generation of hydroperoxides in isolated rat hepatocytes and hepatic mitochondria exposed to hydrophobic bile acids. Gastroenterology 1995;109:1249-56.

2 Galle PR, Theilmann L, Raedsch R, et al. Ursodeoxycholate reduces hepatotoxicity of bile salts in primary human hepatocytes. Hepatology 1990:12:486-91.

3 Guicciardi ME, Gores GJ. Bile acid-mediated hepatocyte apoptosis and cholestatic liver disease. Dig Liver Dis 2002;34:387-92.

4 Yerushalmi B, Dahl R, Devereaux MW, et al. Bile acid-induced rat hepatocyte apoptosis is inhibited by antioxidants and blockers of the mitochondrial permeability transition. Hepatology 2001;33:616-26.

5 Sokol RJ, Straka MS, Dahl R, et al. Role of oxidant stress in the permeability transition induced in rat hepatic mitochondria by hydrophobic bile acids. Pediatr Res 2001;49:519-31.
6 Rodrigues CM, Fan G, Wong PY, et al. Ursodeoxycholic acid may inhibit deoxycholic acid-induced apoptosis by modulating mitochondrial transmembrane potential and reactive oxygen species production. Mol Med 1998;4:165-78.

7 Patel T, Gores GJ. Apoptosis and hepatobiliary disease. Hepatology 1995;21:1725-41.

8 Kurosawa H, Que FG, Roberts LR, et al. Hepatocytes in the bile duct-ligated rat express Bcl-2. Am J Physiol 1997;72:G1587-93.

9 Miyoshi H, Rust C, Guicciardi ME, et al. NF-kappaB is activated in cholestasis and functions to reduce liver injury. Am J Pathol 2001;158:967-75.

10 Patel T, Gores GJ. Inhibition of bile-salt-induced hepatocyte apoptosis by the antioxidant lazaroid U83836E. Toxicol Appl Pharmacol 1997;142:116-22.

11 Muraca M, Fevery J, Blanckaert N. Analytic aspects and clinical interpretation of serum bilirubins. Semin Liver Dis 1988;8:137-47.

12 Ostrow JD, Pascolo L, Tiribelli C. Mechanisms of bilirubin neurotoxicity. Hepatology 2002;35:1277-80.

13 Stocker R, Yamamoto Y, McDonagh AF, et al. Bilirubin is an antioxidant of possible physiological importance. Science 1987;235:1043-6.

14 Stocker R, Glazer AN, Ames BN. Antioxidant activity of albumin-bound bilirubin. Proc Natl Acad Sci U S A 1987:84:5918-22.

15 Tomaro ML, Batlle AM. Bilirubin: its role in cytoprotection against oxidative stress. Int J Biochem Cell Biol 2002;34:216-20.

16 Van Hootegem P, Fevery J, Blanckaert N. Serum bilirubins in hepatobiliary disease: comparison with other liver function tests and changes in the postobstructive period. Hepatology 1985;5:112-17.

17 Ostrow JD, Hammaker L, Schmid R. The preparation of crystalline bilirubinC14. J Clin Invest 1961;40:1442-52.

18 Seglen PO. Preparation of isolated rat liver cells. Methods Cell Biol 1976;1 13:29-83.

19 Vilei MT, Granato A, Ferraresso C, et al. Comparison of pig, human and rat hepatocytes as a source of liver specific metabolic functions in culture systems - implications for use in bioartificial liver devices. Int J Artif Organs 2001;24:392-6.

20 Patel T, Bronk SF, Gores GJ. Increases of intracellular magnesium promote glycodeoxycholate-induced apoptosis in rat hepatocytes. J Clin Invest 1994; $94: 2183-92$

21 Zeid IM, Bronk SF, Fesmier PJ, et al. Cytoprotection by fructose and other ketohesoses during bile salt-induced apoptosis of hepatocytes. Hepatology 1997:25:81-6.

22 Sokol RJ, McKim JM Jr, Goff MC, et al. Vitamin E reduces oxidant injury to mitochondria and hepatotoxicity of intravenous taurochenodeoxycholic acid in the rat. Gastroenterology 1998;114:164-74.

23 Meyer M. Association of serum bilirubin concentration with risk of coronary artery disease. Clin Chem 2000;46:1723-7.

24 Hammerman C, Goldschmidt D, Caplan MS, et al. Protective effect of bilirubin in ischemia-reperfusion injury in the rat intestine. J Pediatr Gastroenterol Nutr 2002;35:344-9.

25 Neale G, Lewis B, Weaver V. Serum bile acids in liver disease. Gut $1971 ; 12: 145-52$.

26 Silva RF, Rodrigues CM, Brites D. Bilirubin-induced apoptosis in cultured rat neural cells is aggravated by chenodeoxycholic acid but prevented by ursodeoxycholic acid. J Hepatol 2001;34:402-8.

27 Rodriguez CM, Fan G, Ma X, et al. A novel role for ursodeoxycholic acid in inhibiting apoptosis by modulating mitochondrial membrane perturbation. J Clin Invest 1998;101:2790-9.

28 Takenaka Y. Bilirubin adsorbent column for plasma perfusion. Ther Apher 1998;2:129-33

29 Pazzi P, Scagliarini R, Puviani AC, et al. Biochemical assessment and clinical evaluation of a non-ionic adsorbent resin in patients with intractable jaundice. Int J Artif Organs 2000;23:312-18.

30 Heemann U, Treichel U, Loock J, et al. Albumin dialysis in cirrhosis with superimposed acute liver injury: a prospective, controlled study. Hepatology 2002;36:949-58. 